\title{
A Theoretical and Experimental Study on Design Creativity
}

\author{
Yong Zeng, Baiquan Yan, Bo Chen, and Shengji Yao \\ Concordia Institute for Information Systems Engineering \\ Concordia University \\ 1455 de Maisonneuve West, CB-410-16 \\ Montreal, Quebec, Canada H3G 1M8 \\ E-mail: zeng@ciise.concordia.ca
}

\begin{abstract}
This paper presents a mathematical model for understanding the factors that lead to a creative design, using the axiomatic theory of design modeling. This mathematical model provides the dynamic mechanism underlying the creative design process: design governing equation. It is pointed out that there may exist a chaotic motion behind the design process because of the nonlinearity of the design governing equation. Based on the recursive-logic based design process model for solving this equation, three routes to creative design are given. A case study from a patent and a software experiment are used to verify the presented mathematical theory.
\end{abstract}

Key Words-design creativity, mathematical model, routes to creative design, axiomatic theory of design modeling, recursive logic

\section{Introduction}

Creative design plays a crucial role in the success of a manufacturing company. However, creativity has been thought of as a mystery and has been attributed to special talents of individual designers. The understanding of design creativity would help the companies to manage and control their product development in a more effective manner. It would also help train creative employees for the companies. This paper aims to study design creativity in a systematic approach.

According to Encyclopedia Britannica, creativity is the ability to make or otherwise bring into existence something new, whether a new solution to a problem, a new method or device, or a new artistic object or form. It can be seen from this definition that a keyword for creativity is "new". The product has to be novel to attribute a design subject creative. In assessing the creativity or novelty of a product, Boden defined two kinds of creativity: psychological and historical [1]. Psychological creativity assumes that the product is novel and valuable at least for the designer. Historical creativity requires that no one else has ever produced such a product before. Though the historical creativity is of more social value, evaluation of the novelty of a design is not our concern in this paper. Instead, we will investigate the mechanism and conditions that lead to creative designs.

To understand the mechanism and conditions leading to creative designs, we need to answer the following questions: what is design creativity? Where does it come from? Is it born or learned? What is the purpose to study it? How does it affect design result? Can design creativity be scientifically understood? In addition to philosophical speculations, two approaches are mainly used to answer these questions: empirical and mathematical.

Empirical approach includes protocol analysis [2], case studies [3], cognitive analysis [4], and retrospective exploration of experienced designers [5]. Based on a protocol analysis, Dorst indicated that creative design is a co-evolution process that looks for problem and solution at the same time [6]. Akin concluded that it is not feasible to search for a unifying theory of the creative process because creativity is domain dependent after studying the creativity in puzzles, inventions and designs [7]. After comparing three cases of creative design in three different domains, Cross concluded that there is a common 'systems approach' to the design problem; but designers may frame their problems in a distinctive and sometimes rather personal manner; and 'first principles' are the foundation of design problem solving [8]. Using the design of microprogramming as an example, Dasgupta points out that inventing is a goal-directed yet opportunistic act; thus the design agent's freedom and capacity to use his knowledge contribute significantly to the design creativity [3]. From the cognitive point of view, Wharton characterized creativity as a focused mental process in which "we are not making something from nothing. Rather, we are taking various existent elements and modifying them such that they interact in a new way" [4].

Mathematical approach attempts to establish a formal model to capture the mechanism of creative design so that the results can be easily adapted to support the development of design tools. Based on the logic of design [9], Zeng speculated that a chaotic motion is implied in design creativity [10]. It was argued that design creativity comes from the interaction of stretching (synthesis) and folding (evaluation) operators in the design process. By stretching, we can find many solutions to the design problem; by folding, we can evaluate those solutions and select the optimal one. This speculation is further supported by the design governing equation proposed by Zeng and $\mathrm{Gu}$ [11]. This design governing equation integrates synthesis and evaluation operators into one function, which renders design problem solving as a process looking for fixed points of this function [12]. However, the form of this equation has been evolving as more robust mathematical tools are being developed [13, 14]. Recently, Kryssanov et al studied creative design using the 
notations of Algebraic Semiotics [15]. They have reached the similar conclusion: the interaction of synthesis and analysis leads to design creativity.

This paper continues to investigate the formal mechanism behind the design creativity. The design process is more naturally connected to the chaotic dynamics through the mathematical operations developed in the axiomatic theory of design modeling [13]. A case study adapted from a patent held by one of the authors is used to illustrate the concepts developed in this paper [16]. A software experiment using finite element mesh generation is used to verify the nonlinear nature of the design process.

\section{A Mathematical Model of Creative Design}

A mathematical model of creative process would be essential and beneficial for developing tools to enhance the design creativity. With the mathematical model, factors affecting the creative design could be better understood. However, the efforts to establish a mathematical model of creative design process have faced fundamental challenges. Creative designing is not a process modifying some parameters of an existing design through numerical methods, but a process full of style, ambiguity, and randomness. It has been argued that there is a fundamental flaw in the mathematical exploration of design activities: if design can be studied mathematically, then randomness in design will disappear and in turn there will be no room for design creativity. This problem was stated by Oxman as [17]: "The knowledge of the precedent is, by definition, of the past. How can it be used not only to explain, but also to generate, the new?" This is equivalent to a classical question in science and philosophy[18]: if natural and human behaviors are governed by deterministic laws, then what is the origin of random behavior and human creativity?

At the turn of last century the French mathematician Poincaré (1929) argued that certain mechanical systems whose time evolution is governed by deterministic law may display chaotic motion: "It may happen that small difference in the initial conditions produce very great ones in the final phenomena." The chaotic motion can be illustrated by a mapping $g: x_{n+1}=g\left(x_{n}\right)$, where an initial difference $\varepsilon$ between two x's is amplified to the separation $\varepsilon e^{n \lambda\left(x_{0}\right)}$, as is shown in Figure 1.

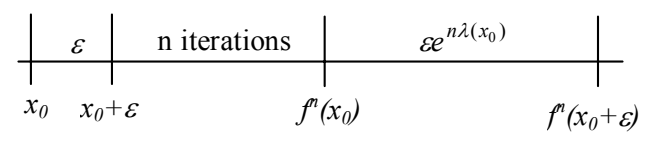

Figure 1. Chaotic motion

It is speculated that the chaotic dynamics underlying the chaotic motion provides a major reason for the randomness of natural behavior. Crutchfield et al[18] argued that "Innate creativity may have an underlying chaotic process that selectively amplifies small fluctuations and models them into macroscopic coherent mental states that are experienced as thoughts. In some cases the thoughts may be decisions, or what are perceived to be the exercise of will. In this light, chaos provides a mechanism that allow for free will within a world governed by deterministic laws." Based on this speculation, the present section aims to identify the possible chaotic motion implied in the design process and its implications to design creativity.

Section 2.1 will first briefly review the theory of dynamic systems, which is the larger framework for chaos. Then the components of design as a dynamical system are defined in 2.2. These components include the state and dynamic of the design process. Section 2.4 presents three routes that may lead to creative designs.

\subsection{Dynamical System and Chaos}

Chaos can be understood from the theory of dynamical systems. A dynamical system consists of two parts: a state and a dynamic [18]. The state is the essential information about the system, the components of which are the coordinates of an abstract construct of a state space. In general the coordinates of the state space vary with the context. For example, the state for a mechanical system might be position and velocity. The state for design will be discussed in the next subsection. The dynamic is a rule used to find how a system evolves from a given initial state with time. The evolution of a system can be visualized in the state space by representing the behavior of the system in geometric form.

A dynamical system is linear if it obeys the superposition rule: the sum of responses to individual stimuli (inputs or initial conditions) equals the single response to the sum of the stimuli. It is nonlinear if it is not linear. Any system that comes into stable motion with the passage of time can be characterized by an attractor in state space, in which rest is an extreme case. Roughly speaking, an attractor is what the behavior of a system settles down to, or is attracted to. Chaos is an attractor that corresponds to unpredictable motions and has a complicated geometric form. There are three basic characteristics of chaos. The first one is an essentially continuous and possibly banded frequency spectrum that resembles random noise. The second one is an ergodicity and mixing of the dynamical orbits which in essence implies the wholesale visit of the entire phase space by the chaotic behavior and a loss of information. The last and most fundamental characteristic of chaos is its sensitive dependence on the initial conditions. A small fluctuation can be amplified in its time evolution, in which the qualitative change occurs to the considered system.

However, not all nonlinear dynamical equations will bring about the chaotic motion. An important element in chaotic dynamics is the existence of a simple stretching and folding operation in the state space. The stretching operation makes an orbit in state space diverge exponentially whereas the folding operation makes the orbit pass close to one another. The orbits on a chaotic attractor are shuffled by these two operators. The randomness of the chaotic orbits is the result of the shuffling process. 


\subsection{Formulation of Design Problem: State of Design}

Design is a recursive process in which design requirements and product descriptions evolve in the same time [9, 11]. This process can be represented in Figure 2. To use the theory of dynamical system in solving a design problem, we should first define the state of design. From Figure 2, the state of design includes two major parts: design requirements and product descriptions.

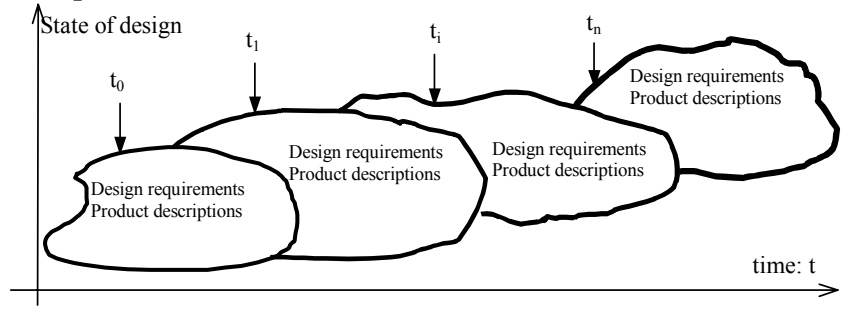

Figure 2. Evolution of the design process

For a design problem in real life, however, there are various types of design requirements and product descriptions at different stages. It is critical to find a base to represent the variety of design. This can be achieved by the notion "engineering system", established in the axiomatic theory of design modeling [13].

An engineering system is the structure of an object $(\Omega)$ including both product (S) and its environment (E).

$\Omega=\mathrm{E} \cup \mathrm{S}, \forall \mathrm{E}, \mathrm{S}$.

Generally speaking, everything except the product itself can be seen as its environment. There are direct, close, and remote environments [19].

Then the engineering system $(\oplus \Omega)$ can be represented as $\oplus \Omega=\oplus(E \cup S)=(\oplus \mathrm{E}) \cup(\oplus \mathrm{S}) \cup(\mathrm{E} \otimes \mathrm{S}) \cup(\mathrm{S} \otimes \mathrm{E})$.

An engineering system can be illustrated in Figure 3. Both $\mathrm{E} \otimes \mathrm{S}$ and $\mathrm{S} \otimes \mathrm{E}$ together are called product-environment boundary. There are two types of product-environment boundary: structural boundary and physical interactions. The structural boundary is the shared physical structure between the product and its environment. The physical interactions include actions $(\mathrm{E} \otimes \mathrm{S})$ of the environment on the product and responses $(\mathrm{S} \otimes \mathrm{E})$ of the product to the environment.

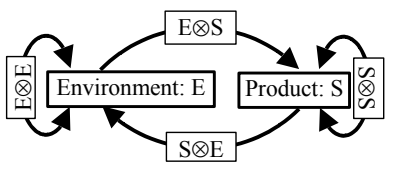

Figure 3. Engineering system

$\oplus \mathrm{E}$ and $\oplus \mathrm{S}$ are structures of the environment and product. Since both product and environment may have components, $\oplus \mathrm{E}$ and $\oplus \mathrm{S}$ can be further decomposed into the structures of these components as well as their mutual relations. This provides a recursive hierarchical representation of design. A more comprehensive study of this recursive hierarchical structure is given in $[13,20]$.

Any information about an engineering system could be derived from or defined based on (2). Obviously, product and product environment are embodied in (2). It has been shown in $[13,21,22]$ that design requirements can also be modeled using (2).

Informally, a design problem can be defined as a proposition in which something has to be designed to meet the descriptions of a request for the design. In this statement, "something to be designed" can be seen as the final design solution of the design problem, which is an engineering system. This engineering system for the final design solution can be formally represented as $\oplus \Omega_{\mathrm{s}}\left(\Omega_{\mathrm{s}}=\mathrm{E}_{\mathrm{s}} \cup \mathrm{S}_{\mathrm{s}}\right)$, where $\mathrm{E}_{\mathrm{s}}$ and $S_{\mathrm{s}}$ are the environment and product by the end of the design process. Similarly, "the descriptions of a request for the design" can also be seen as an engineering system for which the product is not or only partially defined. Denoting the corresponding environment and product by $\mathrm{E}_{0}$ and $\mathrm{S}_{0}$, respectively, we can formally define this engineering system as $\oplus \Omega_{0}\left(\Omega_{0}=\mathrm{E}_{0} \cup \mathrm{S}_{0}\right)$. A design problem can then be symbolically represented as,

$$
\mathrm{P}^{\mathrm{d}}=\lambda\left(\oplus \Omega_{\mathrm{s}}, \oplus \Omega_{0}\right),
$$

where $\lambda$ can be seen as the inclusion relation ( $\supseteq$ ).

In the stage of formulating a design problem, $\oplus \Omega_{\mathrm{s}}$ is an unknown; $\oplus \Omega_{0}$ is the only thing defined. Obviously, $\oplus \Omega_{\mathrm{s}}$ should include all the information in $\oplus \Omega_{0}$. A design problem is solved when $\mathrm{P}^{\mathrm{d}}$ assumes the value of "true". Since $\oplus \Omega_{\mathrm{s}}$ is an unknown in the problem formulation stage, Eq. (3) can be further transformed into the following algebraic form:

$$
\mathrm{P}^{\mathrm{d}}=\mathrm{K}_{\mathrm{e}}\left(\oplus \Omega_{0}\right) \text {, }
$$

where $\mathrm{K}_{\mathrm{e}}$ is called evaluation operator. Its physical meaning will be further discussed later in this paper.

The theorem of design problem structure can be derived from (2) and (3), using the mathematical operations provided by the axiomatic theory of design modeling [13].

$\mathrm{P}^{\mathrm{d}}=\lambda\left(\oplus \mathrm{E}_{\mathrm{s}}, \oplus \mathrm{E}_{0}\right) \wedge \lambda\left(\oplus \mathrm{S}_{\mathrm{s}}, \oplus \mathrm{S}_{0}\right) \wedge \lambda\left(\mathrm{B}_{\mathrm{s}}, \mathrm{B}_{0}\right)$.

Theorem of Design Problem Structure. A design problem is composed of three parts: the environment in which the designed product is expected to work, the requirements on product structure, and the requirements on performances of the designed product.

Based on this theorem, the state of design can be defined by product environment, product description, performance requirements, and structural requirements. They provide a base for representing design problems [13, 21, 22]. These components keep on changing until final design solutions are found to solve the design problem. Hence, the evolution of design can be refined as in Figure 4.

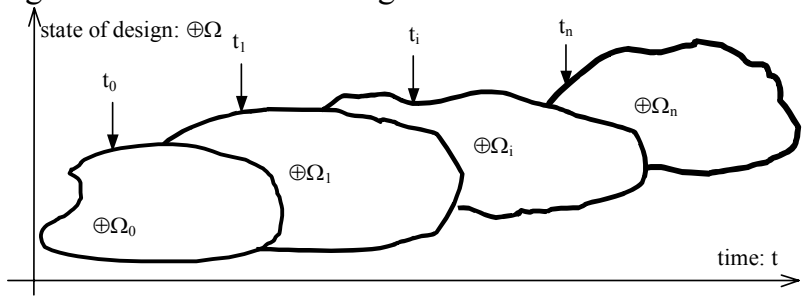

Figure 4. Evolution of the design process: refined

Since the product environment does not change throughout the design process, the following equation can be further 
derived from (3), using the axiomatic theory of design modeling [13, 21].

$$
\begin{aligned}
\mathrm{P}^{\mathrm{d}} & =\lambda\left(\oplus \mathrm{S}_{1}, \oplus \mathrm{S}_{2}\right) \wedge \lambda\left(\mathrm{B}_{1}^{1}, \mathrm{~B}_{2}^{1}\right) \wedge \cdots \wedge \lambda\left(\mathrm{B}_{1}^{\mathrm{n}}, \mathrm{B}_{2}^{\mathrm{n}}\right) \\
& =\mathrm{K}_{\mathrm{e}}^{0}\left(\oplus\left(\mathrm{S}_{0} \cup\left(\bigcup_{\mathrm{j}=1}^{\mathrm{n}} \mathrm{e}_{\mathrm{j}}\right)\right)\right) .
\end{aligned}
$$

This forms the following theorem:

Theorem of Design Problem Decomposition. A design problem can be decomposed in terms of the product environment in which the product is expected to work.

\subsection{Design Governing Equation: Dynamics of Design}

As is requested by the theory of dynamical systems, the dynamic to control the state transition of the system is indispensable for studying the system. As can be seen in Figure 4, each new state of design comes from the design problem defined by its previous state. By applying (4), we have

$$
\mathrm{P}_{\mathrm{i}}^{\mathrm{d}}=\mathrm{K}_{\mathrm{i}}^{\mathrm{e}}\left(\oplus \Omega_{\mathrm{i}}\right),
$$

where $\mathrm{K}_{\mathrm{i}}^{\mathrm{e}}$ is the evaluation operator determined by $\oplus \Omega_{\mathrm{i}}$. $\mathrm{P}_{\mathrm{i}}^{\mathrm{d}}$ represents the current design problem corresponding the state $\oplus \Omega_{\mathrm{i}}$.

On the other hand, a new state of design always comes out of its previous problem definition. This is usually achieved by a synthesis operator $\mathrm{K}_{\mathrm{i}}^{\mathrm{s}}$ so that

$$
\oplus \Omega_{\mathrm{i}+1}=\mathrm{K}_{\mathrm{i}}^{\mathrm{s}}\left(\mathrm{P}_{\mathrm{i}}^{\mathrm{d}}\right) \text {. }
$$

As long as $P_{i}^{d}$ is not true, the design process has to continue.

By substituting (7) into (8), we have

$$
\oplus \Omega_{\mathrm{i}+1}=\mathrm{K}_{\mathrm{i}}^{\mathrm{s}}\left(\mathrm{K}_{\mathrm{i}}^{\mathrm{e}}\left(\oplus \Omega_{\mathrm{i}}\right)\right) \text {. }
$$

We can also substitute (8) into (7),

$$
\mathrm{P}_{\mathrm{i}}^{\mathrm{d}}=\mathrm{K}_{\mathrm{i}}^{\mathrm{e}}\left(\mathrm{K}_{\mathrm{i}}^{\mathrm{s}}\left(\mathrm{P}_{\mathrm{i}-1}^{\mathrm{d}}\right)\right) \text {. }
$$

However, since $\mathrm{P}_{\mathrm{i}}^{\mathrm{d}}$ can always be derived from $\oplus \Omega_{\mathrm{i}}$ according to (7), Eq. (10) is implied in (9). Hence, (9) is sufficient to capture the dynamics of design.

Eq. (9) is called design governing equation. It underlies the design process and governs design activities. It defines the dynamic of design. The basic concept behind this equation is the recursive logic of design [9], which states that design is a recursive process in which the design solution and design problem interdependently evolve $[6,9,23,24]$. The form of this equation has evolved along with the improvement of the formal tool for representing design [11, 12, 14].

Eq. (9) can be further formulated as

$$
\oplus \Omega_{\mathrm{i}+1}=\mathrm{D}^{\mathrm{i}}\left(\oplus \Omega_{\mathrm{i}}\right) \text { where } \mathrm{D}^{\mathrm{i}}=\mathrm{K}_{\mathrm{i}}^{\mathrm{s}} \bullet \mathrm{K}_{\mathrm{i}}^{\mathrm{e}}
$$

Eq. (11) means that design problem solving is a process looking for fixed points of the function $\mathrm{D}^{\mathrm{i}}$. The fixed points for (11) are the interaction points between $\mathrm{y}=\oplus \Omega_{\mathrm{i}}$ and $\mathrm{y}=\mathrm{D}^{\mathrm{i}}\left(\oplus \Omega_{\mathrm{i}}\right)$. A fixed point is usually found through an iterative method. Starting from an initial design state $\oplus \Omega_{0}$, $\oplus \Omega_{1}$ can be found through (11), which updates the design state as well as the function $D^{\mathrm{i}}$. If a sequence of $\oplus \Omega_{\mathrm{j}}$ has a limit $\oplus \Omega_{\mathrm{s}}$, then $\oplus \Omega_{\mathrm{s}}$ is a root of (11). This process is illustrated in Figure 5. In the figure, $D^{0}, D^{1}, \ldots$, and $D^{n}$ are the function in (11) for each stage of the design process. They define different domains for the exploration of design solutions.

Based on (11), the relation between the final solution and the initial problem definition can be represented as

$$
\oplus \Omega_{\mathrm{s}}=\mathrm{D}^{\mathrm{n}} \mathrm{D}^{\mathrm{n}-1} \cdots \mathrm{D}^{0}\left(\oplus \Omega_{0}\right)
$$

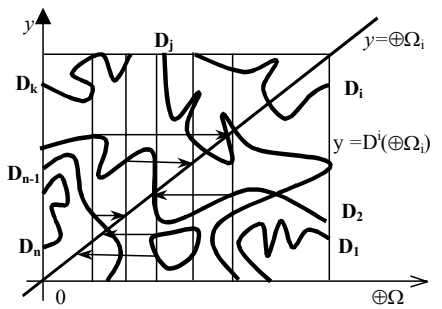

Figure 5. The process looking for design solutions

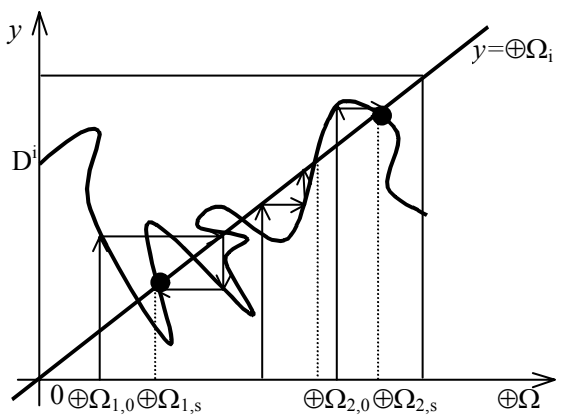

Figure 6. Multiple solutions of a design problem

The design process above is indeed consistent with the recursive logic of design [9]. The evaluation operator $\mathrm{K}_{\mathrm{i}}^{\mathrm{e}}$ is defined in terms of the product structure generated by the synthesis operator $\mathrm{K}_{\mathrm{i}}^{\mathrm{s}}$ in each step of the design process. This fact means that the two operators $\mathrm{K}_{\mathrm{i}}^{\mathrm{e}}$ and $\mathrm{K}_{\mathrm{i}}^{\mathrm{s}}$ are interdependent. Hence, function $\mathrm{D}^{\mathrm{i}}$ is nonlinear. As a result, there may exist multiple fixed points for the same design problem. The convergence depends on the initial design state. This process can be seen in Figure 6 .

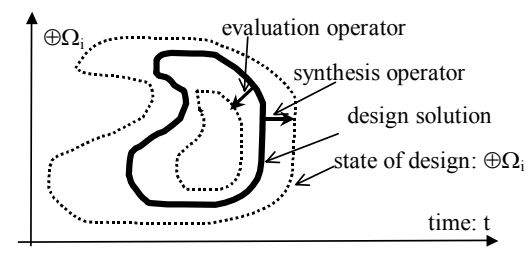

Figure 7. State space of design under synthesis and evaluation operators

The two operators $\mathrm{K}_{\mathrm{i}}^{\mathrm{e}}$ and $\mathrm{K}_{\mathrm{i}}^{\mathrm{s}}$ in (9) correspond to two major phases in the design process: synthesis and evaluation. The synthesis process is responsible for proposing a set of candidate product descriptions based on design requirements. 
It acts like the stretch operator to expand the state space of design. The evaluation process is used to screen candidate products against design requirements. It acts like the folding operator to shrink and adjust the state space of design. The interaction of both operators gives rise to the final balanced design solutions as shown in Figure 7.

Therefore, the design process has an underlying nonlinear dynamics with stretching and folding operators. Chaotic motion may exist in the process. This nonlinear nature will be tested in the software experiment in Section 4.

\subsection{Environment-Decomposition Based Design Process Model: Routes to Creative Design}

2.4.1 Environment-decomposition based design process model The environment-decomposition based design process model can be used to solve the design governing equation [9, $11,13,25]$. This model is illustrated in Figure 8.

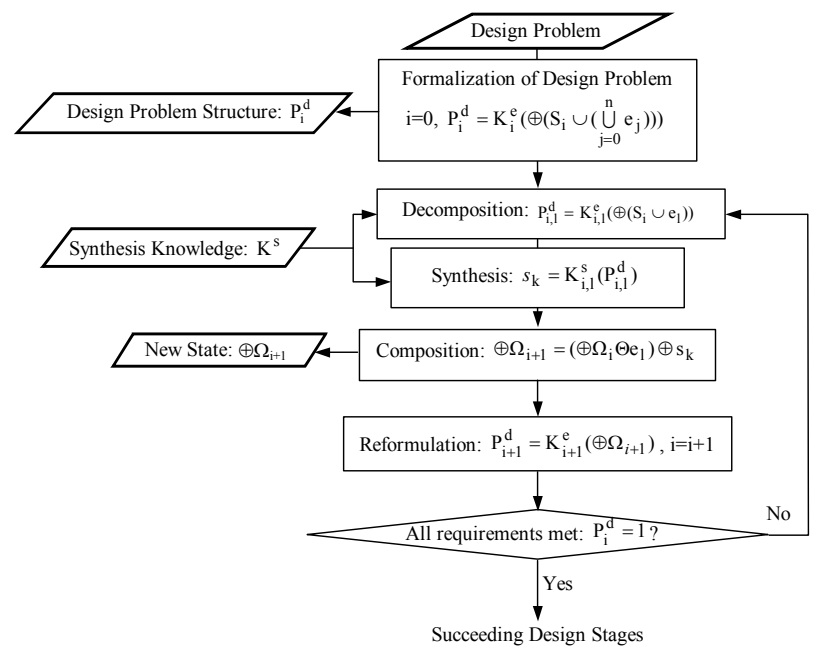

Figure 8. Environment-decomposition based design process model

In this model, a design problem is first formalized into $\mathrm{P}_{\mathrm{i}}^{\mathrm{d}}$ in terms of the product-environment boundary implied in the problem description, on the basis of the theorem of design problem structure. Then according to available synthesis knowledge $\mathrm{K}_{\mathrm{i}, 1}^{\mathrm{s}}$, a primitive part of the product-environment $\mathrm{e}_{1}$ is extracted for generating a primitive product $\mathrm{s}_{1}$, which is then integrated to form an updated design state $\oplus \Omega_{\mathrm{i}+1}$. This process is governed by the theorem of design problem decomposition. Based on this new design state $\oplus \Omega_{\mathrm{i}+1}$, an updated design problem $\mathrm{P}_{\mathrm{i}+1}^{\mathrm{d}}$ can be formulated to evaluate the design state $\oplus \Omega_{\mathrm{i}+1}$ itself. If this evaluation turns out that $\mathrm{P}_{\mathrm{i}+1}^{\mathrm{d}}$ is true, then the design process moves to the next stage. Otherwise the design process continues to decompose the updated product environment implied in $\mathrm{P}_{\mathrm{i}+1}^{\mathrm{d}}$. The key of this model is the simultaneous evolution of design solution and design problem and the solution is to be evaluated in terms of the criteria depending on the solution itself. This is the major difference of this model from the traditional decomposition model. It is also the core idea of the recursive logic [9].
There are three key elements in the environmentdecomposition based design process model: primitive environment, primitive product, and synthesis knowledge [9, $11,25,26]$. These are for the representation of various (maybe infinite) design problems and solutions using finite number of base elements. They are summarized as follow:

1. Primitive environment

$E^{a}=\bigcup_{i=1}^{a_{e}} e_{i}^{a}$

where $\mathrm{e}_{\mathrm{i}}^{\mathrm{a}}$ is a part of primitive environment.

2. Primitive product

$\mathrm{S}^{\mathrm{a}}=\bigcup_{\mathrm{i}=1}^{\mathrm{a}_{\mathrm{s}}} \mathrm{s}_{\mathrm{i}}^{\mathrm{a}}$

where $s_{i}^{a}$ is a part of primitive product.

3. Synthesis knowledge

$\mathrm{K}^{\mathrm{S}}=\mathrm{E}^{\mathrm{a}} \otimes \mathrm{S}^{\mathrm{a}}$

Examples are given in Figure 21.

2.4.2 Three routes to creative design

In the environment-decomposition based design process model, the initial conditions $\mathrm{P}_{\mathrm{i}}^{\mathrm{d}}$ are defined based on the current state of design. This includes two parts: partially completed product structure and the product environment (part of $\oplus \Omega_{\mathrm{i}}$ ). In the design process, however, the partially completed product structure can be taken as a part of environment for the succeeding design. Therefore, in this sense the environment keeps updated even though it is constant before and after the problem is solved. As a result, the ways to update the intermediate environment are essential to get different design solutions, among which creative design may emerge. There are three possible ways to update the environment: first, formulating the design problem differently; second, extending synthesis knowledge; and third, changing the sequence of environment decomposition.

\section{Formulating design problem differently}

Since design is a nonlinear process, the design solution may be dramatically different even if there is only a little difference in the design problem formulation $\mathrm{P}_{0}^{\mathrm{d}}$ according to

Figure 1. This is the basic mechanism for creative design. As can be seen later, other two routes indeed enforce the appearance of this mechanism in the design process. They provide ways to update the design problem in the design process, which continues defines a new design problem based on its current solution. Examples are given in Figure 22 and Figure 23.

\section{Extending synthesis knowledge}

If the synthesis knowledge is extended, then some new and different primitive product $s_{1}$ may be generated for a picked environment part $\mathrm{e}_{1}$. As a result, updated engineering system $\oplus \Omega_{\mathrm{i}+1}$ will be different, which generates a new design problem $\mathrm{P}_{\mathrm{i}+1}^{\mathrm{d}}$. Subsequently, new product structure may be generated. 
The accumulation of this effect in the design process may lead to totally different design solutions.

According to (13), (14), and (15), there are three basic ways to extend the synthesis knowledge: 1) extend the primitive product $S^{a}$;2) extend the primitive environment $E^{a}$; and 3 ) extend the relations between $\mathrm{S}^{\mathrm{a}}$ and $\mathrm{E}^{\mathrm{a}}$.

The third alternative was also observed by Horowitz and Maimon. They presented two sufficient conditions for creative solutions (products): first, no new element needs to be added the solution made use only of existing objects; second, a fundamental relation between two (design) variables is qualitatively changed.

Another situation was identified by Altshuller [5]: creative design arises especially when there is a conflict in the design. Design conflict indeed can be disclosed in the composition process. A conflict adds a new requirement to the design problem formulation. Hence, conflict resolution depends on the available synthesis knowledge. Extension of this knowledge may bring in new possibilities to resolve design creativity and thus lead to creative design.

The difference brought by different synthesis knowledge can be seen in Figure 26.

\section{Changing the sequence of environment decomposition}

It can be seen from Figure 8 that different sequences of environment decomposition may give rise to different reformulation of the design problem because of the application of different synthesis knowledge. As such, the final design solution may be dramatically different. This serves the same purpose as the restructuring of the problem in Akin [27]. It also coincides with a common sense that changing the perspective of seeing a problem may lead to an unusual solution that might be very difficult to identify otherwise. An example is shown in Figure 24 and Figure 25.

\section{Case Study}

The purpose of this case study is to verify the theoretical results from Section 2 through practical product design problem solving. This case study comes from an invention made by one of the authors in extending the lifespan of a light bulb [16]. Two routes to a creative design can be identified in this invention process: different formulation of design problem and extension of synthesis knowledge. A more comprehensive experimental study of design creativity is still ongoing in our research group.

\subsection{Problem}

Incandescent light bulb is one of the most widely used light bulbs. However, incandescent light bulb may burn out easily. Figure 9 illustrates the structure of an Incandescent bulb.

Generally, the failure of a light bulb is caused by the mechanical break in the tungsten filament, because the filament becomes thinner due to the evaporation when the bulb is on. This failure may be accelerated by the following three main factors: 1) mechanical vibration. The hot filament is very fragile, so it will easily burn out if a light bulb is moved or vibrated while it is in service. 2) high line voltage. The high line voltage could greatly shorten the lifespan of a light bulb. 3) the switch's on-and-off. Every time when the switch is turned on, there is an inrush current that applies high thermal stress on the filament. The temperature of the filament may rise to about 2500 Celsius degrees from normal temperature in a very short time. Both the high temperature impact and the thermal stress can increase the evaporation and fatigue of the tungsten filament. Hence, the more often the switch is turned on-and-off the sooner the light bulb will burn out. Figure 10 shows the temperature change of the tungsten filament during the on-and-off circle.

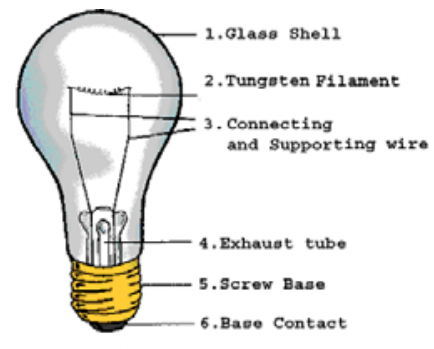

Figure 9. Incandescent light bulb

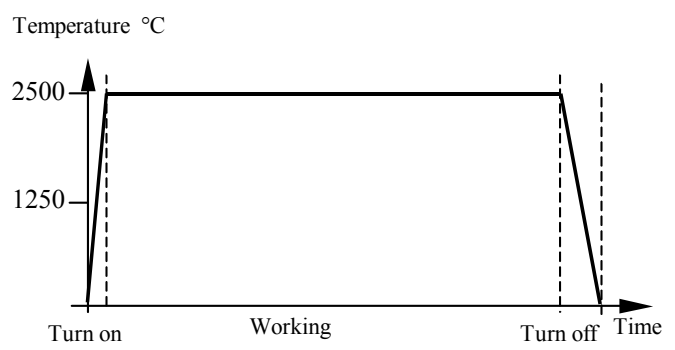

Figure 10. The temperature in the tungsten filament changes during an on-and-off process.

In addition to avoid mechanical vibration and high working voltage, the most effective way to solve this problem is decrease the temperature impact and thermal stress when the light bulb is turned on. We will focus on the strategies to achieve this goal.

\subsection{Different Formulation of the Design Problem}

Figure 11 is a succinct model of this engineering system. As was discussed in 2.2, the state of design is defined by product, environment and their interactions. Therefore, the first step in the problem formulation is to identify the product from environment. This gives rise to different formulation of the design problem.

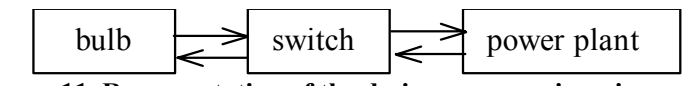

Figure 11. Representation of the design as an engineering system

\subsubsection{Design a new bulb}

The bulb is seen as the product with the switch and power plant being its environment. Other components of the environment include user, market, manufacturing shop, available technologies, etc. Figure 12 shows the diagram of this representation. This formulation will generate a new bulb 
design. This was not a choice of design because of the designer's personal favorite.

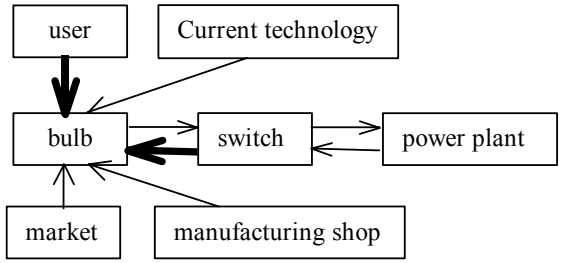

Figure 12. Bulb as the product in the representation of engineering system

\subsubsection{Design a new switch}

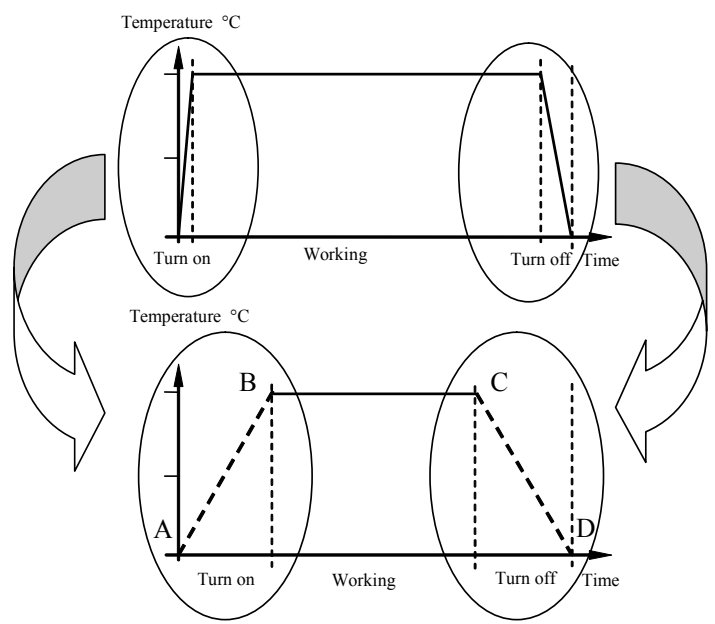

Figure 13. Objective of switch design

Another choice is to design a new switch that can extend the lifespan of a light bulb by delaying the temperature rise in the filament of the bulb. This is illustrated in Figure 13. The dash lines $A B$ and $C D$ indicate that the shape of the curves between $\mathrm{A}, \mathrm{B}$ and $\mathrm{C}, \mathrm{D}$ should be different between different solutions. The following are the requirements for this problem:

1) can decrease the thermal stress and the temperature impact on the light bulb when the switch is turned on;

2) should not lead to hazard and safety problems both to the users and other equipments such as the power line;

3) can be easily operated;

4) should not be much more expensive than ordinary switch;

5) can be manufactured by known manufacturing tools, techniques, and materials.

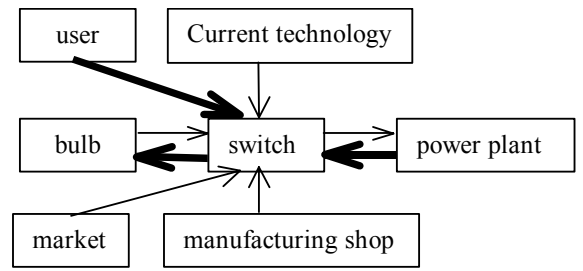

Figure 14. Switch as the product in the representation of engineering system

This problem can then be represented in Figure 14 .
Obviously, the design solution will be totally different from the one focusing on the bulb. Theoretically, we can also redesign the power plant. But it is not practically feasible.

\subsection{Different Synthesis Knowledge}

\subsubsection{Built-in dimmer function}

During the brain storming stage, the inventor tried to find all the methods to achieve the objective illustrated in Figure 13. The first method came into his mind was a dimmer switch, which is able to adjust the brightness of a light bulb. The main electronic component of most dimmer-switches is a TRIAC, which can switch the electricity on and off very quickly. Figure 15 shows the working principle of a TRIAC. In the figure, the vertical lines indicate the points at which a TRIAC is turned on in each half cycle and turned off each time when the voltage equals to zero. That is, when the vertical lines moves from right to left, the bulb should become brighter and brighter. For a dimmer switch, we can control the position of the vertical line by rotating or sliding the switch.

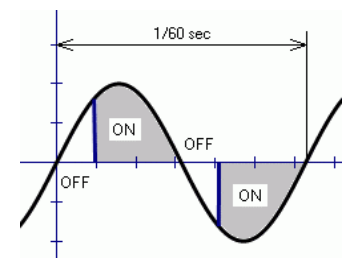

Figure 15. Working principle of a TRIAC

Based on this knowledge, we can design a circuit that can make the vertical line move from right to left automatically when the switch is being turned on.

\subsubsection{SCR circuit}

Figure 16 illustrates another solution. Instead of using a TRIAC, this circuit uses an SCR to delay the turning-on of another half of the cycle. When the switch is being turned on, the bulb will be lit in two steps: being half lit and being fully lit. In this way, the temperature impact and thermal stress will be decreased and the lifespan of the bulb will be prolonged. This circuit can be used either between a switch and the power plant or between a switch and a bulb. Moreover, it can also be combined with an ordinary switch to form a new switch.

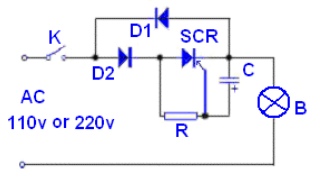

Figure 16. An SCR circuit

\subsubsection{Mechanism and electronic component}

Although the aforementioned two approaches could prolong the life of a light bulb, they also add large amount of extra cost. Therefore, the inventor tried to find a more economic way to solve this problem. Based on the insight gained from those two solutions, the inventor considered the use of a diode to fulfill the objective illustrated in Figure 13.

Diode is a kind of electronic component, which can only allow the electrical current flow in one direction. Figure 17 illustrates the working principle of a diode. Therefore, if a 
diode and a light bulb are cascade connected into an $\mathrm{AC}$ power source, the bulb will be half lit.

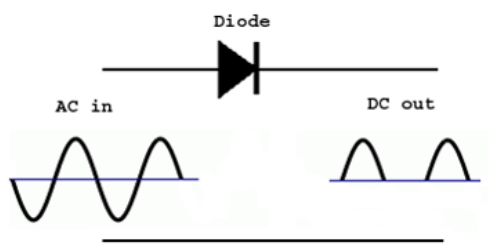

Figure 17. Working principle of a diode

Thus, the problem becomes how to integrate a diode into a switch and make the temperature of the tungsten filament changes according to Figure 18 during an on-and-off cycle.

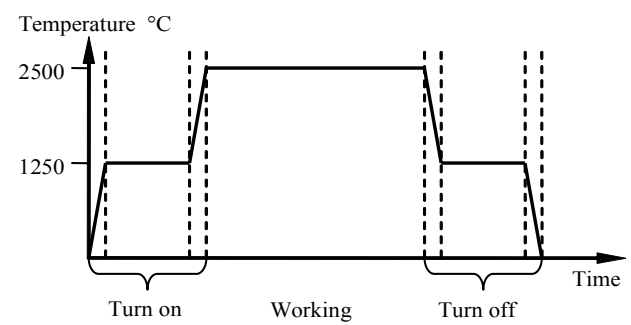

Figure 18. When a bulb is controlled by a switch integrated with diode, the temperature of the tungsten filament changes more smoothly during an on-and-off cycle.

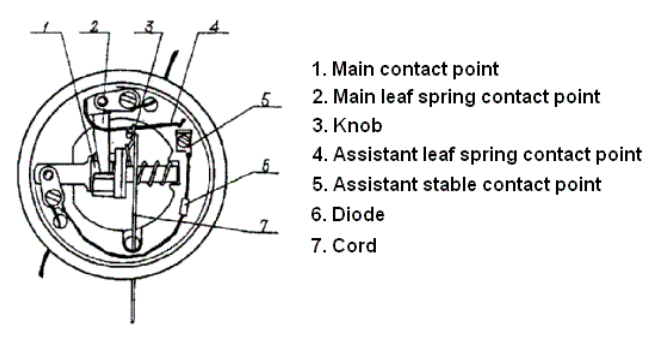

Figure 19. A switch that can extend the life of light bulb

In solving this problem, Chen has made an invention, which only need to add a few very simple and cheap components on an exiting switch [16]. It is illustrated in Figure 19.

When the light bulb is turned on, the switch will be operated in following steps: pull the cord 7 down; the assistant leaf spring contact point 4 will contact with the assistant stable contact point 5 , so the bulb and the diode 6 will be cascade connected; the bulb is then half lit; the knob 3 continually moves downwards; the main contact point 1 will contact with the main leaf spring contact point 2 , then the bulb will be fully lit; release the cord, the knob return to the original position, 4 and 5 are thus disconnected

When the light bulb is turned off, the switch will be operated according to the similar steps: pull the cord 7 down; the assistant leaf spring contact point will contact with the assistant stable contact point 5 ; because the main contact point 1 and the main leaf spring contact point 2 are kept connecting at this time, the bulb is still fully lit; the knob 3 continually move downwards, the main contact point 1 and 2 will be disconnect, the light bulb becomes half lit; release the cord7, the knob return to the original position, 4 and 5 are disconnected, the circuit will be turned off.

\subsubsection{Observations}

It can be seen from the process of this patent generation that different formulations of design problem will generate different design solutions and this formulation also changes during the design process. Moreover, when we deal with a design problem, we could only know the design objectives, but have no idea of the design objects. So it is important to consider a design problem to define the design objects in a "systematic level". (In our case study, the power plant, switch and bulb constitute a system). In many cases even with the same design goal, we may have different design objects used to solve a design problem. By evaluating those design objects, design creativity could be generated. Also, different synthesis knowledge shows different first solution principles. They are also a source of variety of design solutions.

\section{Software Experiment}

The objective of this section is to verify that the recursive logic based design process model implies nonlinear mechanism through a software experiment. The example we use is the finite element mesh design. While this example appears different from most of examples in mechanical design where functionality plays a key role, they are indeed isomorphic to each other [21]. They all consist of four elements: product environment, product descriptions, structural requirements, and performance requirements. For the finite element mesh design problem, the performance requirements come from the accuracy requirement from the later finite element analysis.

This problem aims to generate a quadrilateral mesh for a single-connected plane domain with even number of boundary segments. This mesh needs to satisfy the following requirements: 1) each inner corner $\alpha$ i of a quadrilateral should be within a predefined range (e.g. $45^{\circ} \leq \alpha_{i} \leq 135^{\circ}$ ); 2) the aspect ratio $r_{a}$ (the ratio of opposite edges) and taper ratio $r_{t}$ (the ratio of neighboring edges) of each quadrilateral should be within a predefined range (e.g., $0.1 \leq \mathrm{r}_{\mathrm{a}}, \mathrm{r}_{\mathrm{t}} \leq 10.0$ ); 3 ) the transition from the coarse mesh to the dense mesh should be smooth. In this problem, the product is a quadrilateral mesh. The product-environment boundary is the domain boundary.

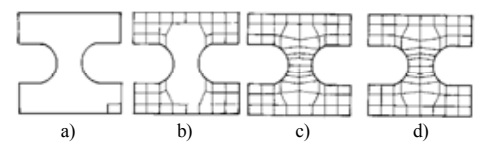

Figure 20. Mesh generation process.

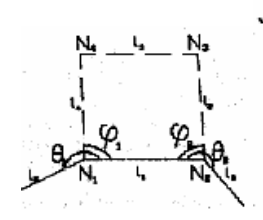

(a)

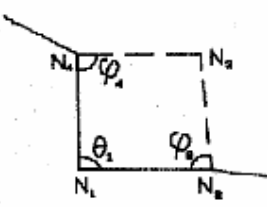

(b)

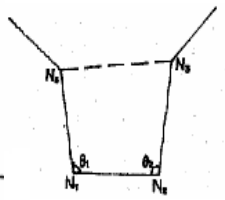

(c)
Figure 21. Synthesis knowledge for generating elements: three boundary configurations are the primitive environment, quadrilateral is the primitive product, and their relations are synthesis knowledge. 
Applying the recursive-logic based design process model, a mesh can be generated element by element through decomposing the product-environment boundary. Figure 20 is an example of this process. In generating an element, one of three synthesis rules is used. These rules are shown in Figure 21. For the first rule, the best element would be a square whereas the best element for the second rule would be the ones with rectangular $\varphi_{2}$ and $\varphi_{4}$. However, since the productenvironment boundary is updated every time a new element is generated, each element should be generated in such a way that the remaining boundary is still good enough for the mesh design. These two aspects correspond to the stretching and folding operations of design. The following factors determine the final mesh: 1) formulation of design problem; 2) strategy of problem decomposition; 3) basic synthesis rules. We will show how small change in these factors may dramatically affect the final solutions.

\subsection{Formulation of Design Problem.}

The formulation of the mesh design problem lies in the definition of boundary segments and all design requirements. Figure 22 shows that a small change of the coordinate of one point results in different mesh topologies.

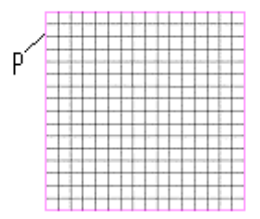

Solution 1

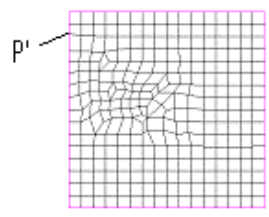

Solution 2
Figure 22. Small change of one boundary segment leads to different mesh topology

The difference is shown in the following table.

\begin{tabular}{|c|c|}
\hline Corresponding points & $(\mathrm{x}, \mathrm{y})$ \\
\hline Point $\mathrm{p}$ in solution 1 & $(0,-0.25)$ \\
\hline Point $\mathrm{p}^{\prime}$ in solution 2 & $(0,-0.225)$ \\
\hline
\end{tabular}

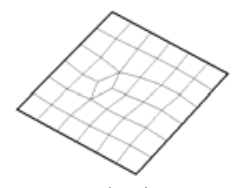

Solution 1

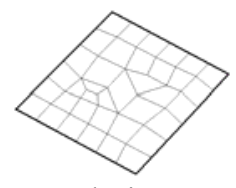

Solution 2

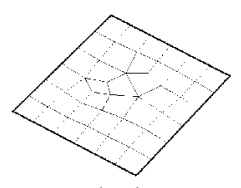

Solution 3
Figure 23. Small change in design requirement leads to different mesh topology

Figure 23 gives three solutions coming from small differences in their requirement definition. All requirements are the same except for inner corners. In solution 1, the requirement for the inner corner of an element is between $43^{\circ}$ and $145^{\circ}$ while in solution 2 is between $45^{\circ}$ and $145^{\circ}$ and in solution 3 the inner corner is between $48^{\circ}$ and $145^{\circ}$.

\subsection{Strategy of Design Problem Decomposition}

The decomposition of this mesh design problem lies in the selection of the point around which an element is going to be generated. This means designing from different perspectives.

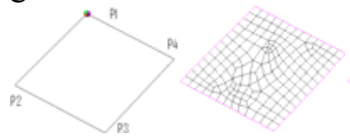

(a) (b)

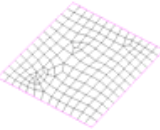

(c)

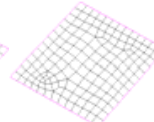

(d)

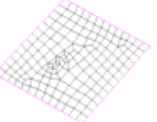

(e)
Figure 24. Different starting point results in different solutions

Figure 24 shows four results generated with the same boundary but with different starting point. The boundary is shown in Figure 24a). Results in Figure 24b, c, d, e) are the mesh created by selecting point $\mathrm{p} 1, \mathrm{p} 2, \mathrm{p} 3$, and $\mathrm{p} 4$ as the starting point, respectively. Obviously, the four solution's topologies are totally different.

Figure 25 shows that different method of updating the boundary yield different solutions. In method (a), when to select a reference point, the boundary list is searched from the point immediately following the former reference point; in method (b), always from the beginning; and in method (c), from a random position. These three methods for selecting the current point represent three different methods of problem reformulation.

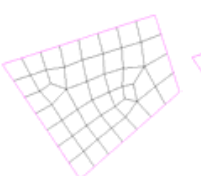

(a)

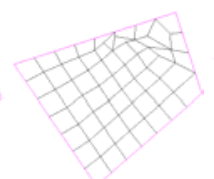

(b)

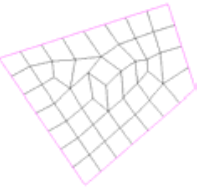

(c)
Figure 25. Different strategies for updating boundary points

\subsection{Extension of Basic Synthesis Rules}

As shown in Figure 21, there are three basic geometric rules for generating a quadrilateral mesh. Based on rule b, and c, five equivalent algebraic rules are created. To make the problem simple, we just name these five rules as rule 1, rule 2, $\ldots$ rule 5 . Actually, the five rules represent basic design knowledge for this design problem. Figure 25 shows some solutions generated with different rules (design knowledge).

Solution a was created using all the five rules, solution $b$ was created using only rule 5 , and solution $\mathrm{c}$ was created using rule 1 and rule 2 together.

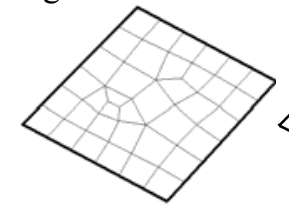

(a)

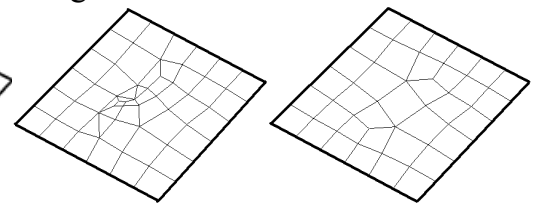

(b) (c)
Figure 26. Effects of synthesis knowledge on solutions

From the results, it is easy to see that when different rules are used, the solution is different. This indicates that a designer's knowledge plays a role in design activity. Generally, the more the knowledge a designer has, the better the solution a designer would create. However, the results show that a large quantity of design knowledge doesn't mean a creative solution will follow; the strategy to use the design knowledge, to a remarkable extent, is more important than knowledge itself in creative design. 


\subsection{Observations}

Through the examples given above, it can be seen that there is a nonlinear mechanism behind the recursive-logic based design process model. This makes a small difference in problem definition, problem decomposition, and synthesis rules exemplified in the final design solutions. This sensitivity to initial conditions is a characteristic of creative design as is illustrated in Figure 1.

Though initial conditions have strong effects on the design solutions, it is not the case that this will always happen to design. Under some conditions, even big changes in the initial conditions will not make much difference. This may happen to the region where only one design solution is available.

Though the example used in this section is not a typical product design problem, it has the isomorphic structure. A computer-aided conceptual product design system is under development by applying the same principles.

\section{Conclusion}

This paper has proposed a formal mechanism of creative design. This mechanism is based on design governing equation, which relates the design process to the chaotic motion. Design solutions are found by looking for the fix points of the design governing equation. Both theoretical and experimental studies show that the design process is a nonlinear, dynamic and recursive process. Three routes to a creative design are also developed based on the sensitivity of final solutions to the initial conditions for a nonlinear dynamic system. A case study based on a patent held by one of the authors is used to illustrate the concepts proposed in this paper. A finite element mesh design program is used to verify the nonlinear nature of the design process.

\section{Acknowledgment}

This work is partially supported by NSERC (Grant number RGPIN 298255).

\section{References}

[1] Boden, M., 1990, The Creative Mind, Weidenfeld and Nicolson, London.

[2] Liu, Y.-T., 2000, "Creativity or Novelty?: Cognitive-Computational Versus Social-Cultural," Design Studies, 21(3), pp. 261-276.

[3] Dasgupta, 1994, Creativity in Innovation and Design.

[4] Wharton, J. G.,1999, Cognition and Creativity: Creativity, Imagination and the Objectivist Epistemology, www.axiomatic.net/cc.pdf

[5] Altshuller, G. S., 1984, Creativity as an Exact Science: The Theory of the Solutions of Inventive Problems, Gordon and Breach Science Publishers.

[6] Dorst, K. and Cross, N., 2001, "Creativity in the Design Process: CoEvolution of Problem-Solution," Design Studies, 22(5), pp. 425-437.

[7] Akin, O. and Akin, C., 1998, "On the Process of Creativity in Puzzles, Inventions, and Designs," Automation in Construction, 7, pp. 123-138.

[8] Cross, N., 1997, "Descriptive Models of Creative Design: Application to an Example," Design Studies, 18(4), pp. 427-440.
[9] Zeng, Y. and Cheng, G., 1991, "On the Logic of Design," Design Studies, 12(3), pp. 137-141.

[10] Zeng, Y., Knowledge-Based Finite Element Model Design: Theory and Implementations, in Research Institute of Engineering Mechanics. 1992, Dalian University of Technology: Dalian. pp. 109

[11] Zeng, Y. and Gu, P., 1999, "A Science-Based Approach to Product Design Theory Part I: Formulation and Formalization of Design Process," Robotics and Computer-Integrated Manufacturing, 15(4), pp. 331-339.

[12] Zeng, Y. and Gu, P., 1999, "General Design Governing Equation," NSFC Grantee's Conference on Design and Manufacturing Engineering, Vancouvar, BC, Canada.

[13] Zeng, Y., 2002, "Axiomatic Theory of Design Modeling," Transaction of SDPS: Journal of Integrated Design and Process Science, 6(3), pp. 1-28.

[14] Zeng, Y., An Axiomatic Approach to the Modeling of Conceptual Product Design Using Set Theory, in Department of Mechanical and Manufacturing Engineering. 2001, University of Calgary: Calgary. pp. 218

[15] Kryssanov, V. V., Tamaki, H. and Kitamura, S., 2001, "Understanding Design Fundamentals: How Synthesis and Analysis Drive Creativity, Resulting in Emergence*1," Artificial Intelligence in Engineering, 15(4), pp. 329-342.

[16] Chen, B., A Switch That Can Extend the Life of Light Bulb, in State Intellectual Property Office. 1998: People's Republic of China

[17] Oxman, R., 1990, "Prior Knowledge in Design: A Dynamic Knowledge-Based Model of Design and Creativity," Design Studies, 11(1), pp. 17-28.

[18] Crutchfield, J. P. e. a., 1986, "Chaos," Scientific American, 255(6), pp. 38 49.

[19] Hubka, V. and Eder, W., 1988, Theory of Technical Systems, SpringerVerlag.

[20] Zeng, Y., Pardasani, A., Antunes, H., Li, Z., Dickinson, J., Gupta, V. and Baulier, D., 2004, "Mathematical Foundation for Modeling Conceptual Design Sketches," Transactions of the ASME: Journal of Computing and Information Science in Engineering, 4(2).

[21] Zeng, Y., 2004, "Mathematical Formulation of Design Problems," Journal of Engineering Design, submitted.

[22] Zeng, Y., 2003, "Formalization of Design Requirements," Integrated Design and Process Technologies, IDPT-2003.

[23] Maher, M. and Tang, H., 2003, "Co-Evolution as a Computational and Cognitive Model of Design," Research in Engineering Design, 14, pp. 47-63.

[24] Zeng, Y. and Cheng, G., 1993, "Knowledge Based Free Mesh Generation of Quadrilateral Elements in Two Dimensional Domains," Microcomputers in Civil Engineering, 8(4), pp. 259-270.

[25] Zeng, Y. and Gu, P., 2001, "An Environment Decomposition-Based Approach to Design Concept Generation," Proceedings of International Conference on Engineering Design'01, pp. 525-532.

[26] Zeng, Y. and Gu, P., 1999, "A Science-Based Approach to Product Design Theory Part Ii: Formulation of Design Requirements and Products," Robotics and Computer Integrated Manufacturing, 14(4), pp. 341-352.

[27] Akin, O., 1990, "Necessary Conditions for Design Expertise and Creativity," Design Studies, 11(2), pp. 107-113. 